\title{
Feasibility and Efficacy of Accelerated Weekly Concomitant Boost Postoperative Radiation Therapy Combined with Concomitant Chemotherapy in Patients with Locally Advanced Head and Neck Cancer
}

\author{
Berrin Pehlivan, $\mathrm{MD}^{1}$, Francois Luthi, $\mathrm{MD}^{2}$, Oscar Matzinger, $\mathrm{MD}^{1}$, Michael Betz, $\mathrm{MD}^{1}$, Daniela Dragusanu, $\mathrm{MD}^{1}$, \\ Shelley Bulling, $\mathrm{MSc}^{1}$, Luc Bron, $\mathrm{MD}^{3}$, Philippe Pasche, $\mathrm{MD}^{3}$, Walter Seelentag, $\mathrm{MD}^{4}$, René O. Mirimanoff, $\mathrm{MD}^{1}$, \\ Abderrahim Zouhair, $\mathrm{MD}^{1}$, and Mahmut Ozsahin, $\mathrm{MD}, \mathrm{PhD}^{1}$ \\ ${ }^{1}$ Department of Radiation Oncology, University Hospital Center, University of Lausanne, Lausanne, Switzerland; \\ ${ }^{2}$ Department of Medical Oncology, University Hospital Center, University of Lausanne, Lausanne, Switzerland; \\ ${ }^{3}$ Department of Otorhinolaryngology, University Hospital Center, University of Lausanne, Lausanne, Switzerland; \\ ${ }^{4}$ Department of Pathology, University Hospital Center, University of Lausanne, Lausanne, Switzerland
}

Background. The aim of this study was to assess feasibility and efficacy of weekly concomitant boost accelerated postoperative radiation therapy (PORT) with concomitant chemotherapy (CT) in patients with locally advanced head and neck cancer (LAHNC).

Methods and Materials. Conformal or intensity-modulated 66-Gy RT was performed in 5.5 weeks in 40 patients. Cisplatin was given at days 1, 22, and 43. Median followup was 36 months.

Results and Discussion. Grade 3 mucositis, dysphagia, and erythema was observed in ten (25\%), nine (23\%), and six $(13 \%)$ patients, respectively. Grade 3 or more anemia was observed in two $(6 \%)$ patients, and leukopenia in five $(13 \%)$ patients. No grade 3 or 4 thrombocytopenia was observed. Grade 3 nephrotoxicity was observed in one patient (3\%). No treatment-related mortality was observed.

Abderrahim Zouhair and Mahmut Ozsahin contributed equally as senior author.

Presented in part at the 14th European Cancer Organisation (ECCO), Barcelona, Spain, 23-27 September 2007; and at the 49th Annual Meeting of the American Society for Therapeutic Radiology and Oncology (ASTRO), Los Angeles, CA, USA, 28 October-1 November 2007.

(C) Society of Surgical Oncology 2009

First Received: 22 December 2008;

Published Online: 12 March 2009

M. Ozsahin, $\mathrm{MD}, \mathrm{PhD}$

e-mail: mahmut.ozsahin@chuv.ch
Grade 2 or more xerostomia and edema were observed in ten $(25 \%)$ and one (3\%) patient, respectively. Locoregional relapse occurred in eight patients, and seven patients developed distant metastases. Median time to locoregional relapse was 6 months. Three-year overall, disease-free survival, and locoregional control rates were $63 \%, 62 \%$, and $81 \%$, respectively. Multivariate analysis revealed that the only prognostic factor was nodal status.

Conclusion. Reducing overall treatment time using accelerated PORT/CT by weekly concomitant boost (six fractions per week) combined with concomitant cisplatin $\mathrm{CT}$ is easily feasible with acceptable morbidity.

Following surgery, postoperative radiotherapy (RT) combined with concomitant chemotherapy (CT) improves the outcome in locally advanced head and neck cancer (LAHNC). ${ }^{1,2}$ Despite multimodality treatment, locoregional relapse risk remains as high as $35-40 \% .^{3-5}$ The risk of recurrence or metastasis is particularly high in patients with inadequate resection margins, extranodal spread or multiple involved lymph nodes. ${ }^{6}$ Various strategies have been proposed to improve the outcome. ${ }^{7-10}$ Two major approaches have gained importance in recent years to improve the outcomes: altered fractionation and concomitant CT regimens. Confirming expectations based on radiobiological theory, altered fractionation regimens could overcome accelerated tumor population, and concurrent delivery of CT and ionizing radiation could sensitize tumor clonogens. 
Data concerning hyperfractionated or accelerated RT with concomitant CT are very limited, and there are no prospective data in the postoperative setting. We previously showed the feasibility of accelerated (six fractions/week) fractionated 66-Gy postoperativer RT (PORT) using a single-fraction regimen from Monday to Thursday and a concomitant boost (two fractions separated by 6-h interval) at Friday afternoon sessions with acceptable toxicity. ${ }^{11}$ On the basis of these findings, in this study we combined concomitant cisplatin CT to accelerated PORT in patients with LAHNC in order to increase locoregional control (LRC) and survival.

\section{METHODS AND MATERIALS}

Forty consecutive patients with advanced-stage squamous-cell carcinoma of head and neck treated at the University Hospital of Lausanne (CHUV) were included in this study. All patients were treated with curative surgery followed by accelerated PORT and concomitant CT. Inclusion criteria consisted of nonmetastatic LAHNC classified as pT1-pT4 and/or pN0-pN3, age 18 years or older, no previous history of cancer other than nonmelanoma skin cancer or in situ cervix cancer, and good performance status [World Health Organization (WHO) scale $0-1]$. The study was approved by the Ethical Committee of the University of Lausanne. T- and $\mathrm{N}$ classification were assigned according to the staging system of the International Union against Cancer (UICC) 2002. ${ }^{12}$ Pretreatment characteristics are listed in Table 1.

Pretreatment evaluation included a medical history, examination with panendoscopy (oro-pharyngo-laryngoscopy, bronchoscopy, and esophagoscopy), and computed tomography and/or magnetic resonance imaging (MRI) of the head and neck region in all cases. Additional diagnostic procedures for distant metastases, including computed tomography of the chest, liver ultrasound, bone scintigraphy, and/or more recently positron emission tomography, were only performed if clinically indicated. Laboratory studies included blood chemistry (electrolytes, liver and kidney function tests), and complete blood count were performed each week of the treatment.

All patients were seen and discussed at our multidisciplinary tumor board, and the treatment recommendation was made jointly by head and neck surgeons, radiation oncologists, and medical oncologists with advice from the diagnostic radiologist, pathologist, nuclear medicine specialist, nursing staff, nutritionists, and dentists. Percutaneous endoscopic gastrostomy (PEG) was placed before or at the beginning of treatment in 17 of the $40(43 \%)$ patients, and 4 patients $(10 \%)$ had nasogastric feeding tube on the basis of our previous experience. ${ }^{13}$ Nutritional intervention, professional surveillance, advice, and support
TABLE 1 Patient characteristics

\begin{tabular}{|c|c|c|}
\hline & Number & $\%$ \\
\hline \multicolumn{3}{|l|}{ Tumor site } \\
\hline Oral cavity & 15 & 38 \\
\hline Oropharynx & 8 & 20 \\
\hline Hypopharynx & 8 & 20 \\
\hline Larynx & 7 & 18 \\
\hline Unknown primary & 2 & 5 \\
\hline \multicolumn{3}{|l|}{$R T$ indications } \\
\hline Extranodal infiltration: & 24 & 60 \\
\hline With $(+)$ surgical margin & 13 & 32 \\
\hline Without $(+)$ surgical margin & 11 & 28 \\
\hline Without extranodal infiltration & 16 & 40 \\
\hline$(+)$ surgical margin & 9 & 22 \\
\hline T4 tumors & 5 & 13 \\
\hline$\geq 3$ lymph nodes & 2 & 5 \\
\hline \multicolumn{3}{|l|}{$p T$-classification } \\
\hline 0 & 2 & 5 \\
\hline 1 & 5 & 12 \\
\hline 2 & 16 & 40 \\
\hline 3 & 2 & 5 \\
\hline 4 & 15 & 38 \\
\hline \multicolumn{3}{|l|}{$p N$-classification } \\
\hline 0 & 3 & 7 \\
\hline 1 & 8 & 20 \\
\hline $2 \mathrm{a}$ & 2 & 5 \\
\hline $2 b$ & 15 & 38 \\
\hline $2 c$ & 5 & 12 \\
\hline 3 & 7 & 18 \\
\hline
\end{tabular}

were available during the entire treatment period; prophylactic mouth washes were recommended, and fungal or bacterial infections on mucous membranes were treated according to microbiologic testing. All patients had dental examination and, if necessary, dental treatment before start of treatment.

\section{Postoperative Treatment}

Postoperative accelerated 66-Gy RT was given using six fractions per week (2 Gy/fraction) with a single-fraction regimen from Monday to Thursday and a two fractions including a concomitant boost on Friday (with an interval of at least $6 \mathrm{~h}$ ) during 5.5 weeks (38 days). RT was implemented in all patients using five-field three-dimensional (3D) conformal or intensity-modulated RT (IMRT), according to the Groupe Oncologie-Radiothérapie Tête et Cou (GORTEC)/European Organisation for Research and Treatment of Cancer (EORTC)/Radiation Therapy Oncology Group (RTOG) guidelines. The boost volume (66 Gy) 
consisted of the clinical target volume (CTV1), which was defined according to the presurgical location of the primary tumor, and its nodal extension including the entire surgical bed. Planning target volume (PTV1) included a 5-mm margin around the CTV1 in three dimensions. The initial treatment volume (PTV2, 46-50 Gy) consisted of the boost volume and all nodal areas at risk of subclinical malignant disease (PTV2), including a 5-mm margin around the CTV2 in three dimensions. Patient immobilization was realized using individualized thermoplastic immobilization masks in all patients.

Concomitant cisplatin CT was planned at $100 \mathrm{mg} / \mathrm{m}^{2}$ at days 1,22 , and 43 in all but five patients, in whom carboplatin [area under the curve $(\mathrm{AUC})=2$ weekly, or $\mathrm{AUC}=5$ days 1, 22, and 43] was chosen due to inadequate renal function.

Patients received prophylactic hydration and antiemetic agents and were seen weekly by the radiation oncologist, head and neck surgeon, and/or medical oncologist during RT. Special advice and attention were given by the nursing team to skin care, and oral hygiene was enforced. Symptomatic treatment for mucositis (sodium bicarbonate) was offered, and oral candidiasis was promptly treated by fluconazol.

\section{Toxicity Assessment and Follow-Up}

Toxicity assesment was done according to the Common Toxicity Criteria (CTC)/National Cancer Institute (NCI) v3.0. Patients were not assessed by the same observer. Clinical examination and toxicity assessment was repeated weekly during RT. Patients were then followed by the head and neck surgeon and radiation oncologist weekly during the first 2 months, monthly assessments from 2-6 months, and every 2-3 months until 2 years. At that time, a panendoscopy was performed, and MRI, computed tomography, and biopsies were performed as indicated. Patients without evidence of disease were subsequently followed every 6 months up to 5 years, then once per year.

\section{Statistical Methods}

Cause-specific survival (CSS), disease-free survival (DFS), and overall survival (OS) rates as well as actuarial LRC rates were calculated using product-limit method. ${ }^{14}$ Time to any event was measured from the date of pathological diagnosis. The events were death (all causes) for OS, head and neck cancer-related mortality for CSS, and death (all causes) or relapse for DFS. For the LRC rate, the event consisted of local or regional relapse. Confidence intervals (CI) were calculated from standard errors. Differences between groups were assessed using the log-rank test. $^{15}$ Multivariate analyses were done using the Cox stepwise-regression analysis to determine the independent contribution of each prognostic factor. ${ }^{16}$

\section{RESULTS}

Between March 2001 and April 2006, 36 men and 4 women with pT1-pT4 and/or pN0-pN3 LAHNC were included in this study. Median age was 60 years (range 3681 years). Indications of PORT/CT were positive surgical margins ( $n=9$; all R1), T4 R0 tumors $(n=5)$, three or more positive lymph nodes without extranodal infiltration (all R0; $n=2$ ) in $16(40 \%)$ patients; or extranodal infiltration with (all R1; $n=13$ ) or without $(n=11)$ positive surgical margins in $24(60 \%)$ patients. Twenty-three patients with T0 $(n=2)$, T1 $(n=5)$, and T2 $(n=16)$ were included because of positive surgical margins $(n=4$; all R1) or because of advanced nodal disease.

Median interval between surgery and RT was 46 days (range 24-112 days), and median RT duration was 39 days (range 35-62 days). In five patients, treatment duration was longer than 45 days because of toxicity. All but two patients received the planned total dose without unplanned interruption (66 Gy in 38, $64 \mathrm{~Gy}$ in 1, and $58 \mathrm{~Gy}$ in 1). Three cycles of planned full-dose cisplatin could be administered in 18 patients $(54 \%)$. Because of toxicity, 15 patients $(43 \%)$ could receive only two cycles of CT, and 2 patients only $(3 \%)$ one cycle of CT. Dose reduction of cisplatin was necessary in two patients, who could receive only two cycles of CT. Carboplatin was given weekly in one patient, and every 3 weeks in four patients.

Three patients had local relapse only, one patient locoregional relapse, two patients regional relapse alone, two patients regional relapse and distant metastases, and five patients had only distant metastases (Fig. 1). Median time to locoregional relapse was 6 months (range 1-40 months). Three-year OS, CSS, DFS, and LRC rates were 63\% $(95 \%$ CI $46-78 \%$ ), 66\% (95\% CI 50-82\%), 62\% (95\% CI 48-

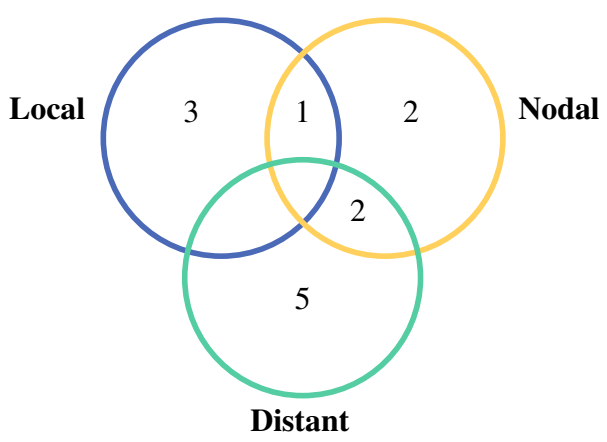

FIG. 1 Distribution of relapses in 40 patients treated with accelerated weekly concomitant boost postoperative radiation therapy combined with concomitant chemotherapy 


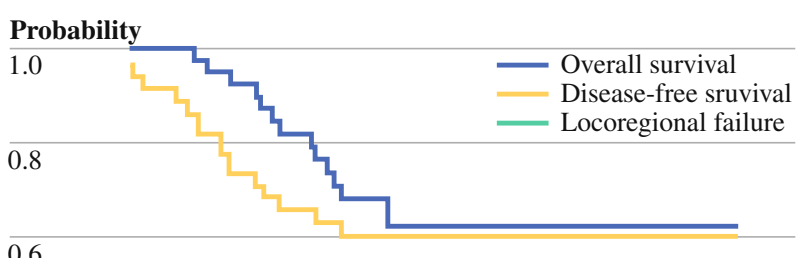

0.6

0.4

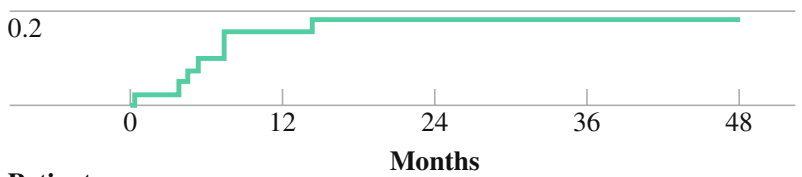

Patients at Risk

\begin{tabular}{llllll}
\hline & 40 & 30 & 22 & 14 & 8 \\
\hline$\square$ & 40 & 27 & 22 & 14 & 8 \\
\hline & 40 & 33 & 22 & 14 & 8 \\
\hline
\end{tabular}

FIG. 2 Probability of overall survival (solid line), disease-free survival (dotted line), and locoregional failure (dashed line) in 40 patients treated with accelerated weekly concomitant boost postoperative radiation therapy combined with concomitant chemotherapy

78\%), and 81\% (95\% CI: 69-93\%), respectively (Fig. 2). Distant metastasis probability at 3 years was $20 \%$ (95\% CI 7-33\%). For univariate analyses gender, age, tumor site, pT- classification, pN-classification, extracapsular nodal extension, positive surgical margins, interval between surgery and RT, and total RT time were analyzed (Table 2). Univariate and multivariate analyses revealed that the only prognostic factor influencing the outcome was nodal status, for both DFS and LRC [relative risk $(\mathrm{RR})=0.40, P=0.02$; and $\mathrm{RR}=0.0004, P=0.02]$.

Incidence of grade 3 mucositis was $25 \%(n=10)$, grade 3 dysphagia $23 \%(n=9)$, and grade 3 skin erythema $13 \%$ $(n=5)$. CT-related anemia was observed in two patients (grade 3 in one, and grade 4 in one), leukopenia in four patients (grade 3 in two, and grade 4 in two), and no grade 3 or 4 thrombopenia was observed. Grade 3 renal toxicity was observed in only one patient (Table 3). Median weight loss was $3.5 \mathrm{~kg}$ (range $0-14.5 \mathrm{~kg}$ ). No treatment-related mortality was observed.

Considering late effects, grade $0,1,2$ or 3 xerostomia was observed in $9(23 \%), 22(55 \%), 9(23 \%)$, and $1(3 \%)$ patient, respectively; grade 0,1 , and 2 edema was observed in $25(63 \%), 14(35 \%)$, and $1(3 \%)$ patient, respectively. Two patients developed mandibular osteoradionecrosis.

\section{DISCUSSION}

The impact of shortening overall treatment time on therapeutic outcome is well established. Several studies validated that, compared with conventional fractionation using 2 Gy per day, altered fractionation (especially hyperfractionated or accelerated RT) increases locoregional tumor control in patients with HNSCC. ${ }^{9,17-19}$ Ang et al. compared an accelerated concomitant boost PORT delivering a total dose of 63 Gy in 35 fractions of 1.8 Gy during 5 weeks (five fractions/week during 3 weeks and two fractions/day during 2 weeks) with a conventionally fractionated PORT delivering a total dose of $63 \mathrm{~Gy}$ in 35 fractions of $1.8 \mathrm{~Gy} /$ fraction during 7 weeks. ${ }^{20}$ This study, in the accelerated PORT arm, showed higher but not statistically significant improvement in 5-year LRC $(P=0.11)$ and $\mathrm{OS}(P=0.08)$ in favor of accelerated arm. However, it confirmed prospectively that, in patients treated by conventional fractionation, delay of more than 6 weeks between surgery and RT significantly reduces LRC and OS. Another important study by Overgaard et al. compared a modestly accelerated regimen of $66-70$ Gy in 2-Gy fractions given six times per week with the same dose given in five fractions per week, using nimorazole in both groups. $^{21}$ They reported a gain of $15 \%$ in local control using accelerated RT, with increased acute toxicity but no increase in late toxicity. In the postoperative setting, Sanguineti et al. and Swunski et al. confirmed the feasibility of accelerated PORT. ${ }^{22,23}$ Like Ang et al., Sanguineti et al. found that patients delaying the start of RT benefited more from accelerated RT in terms of LRC; however, they did not observe any difference between the standard and accelerated treatment arms (2-year LRC of $80 \%$ and $78 \%$, respectively). Swunski et al. reported acceptable acute toxicity, in spite of high intensity and duration of mucosal reactions, in the continous accelerated treatment arm.

The impact of overall treatment time on outcome is more important for locoregional control than survival. Overall, the incidence of distant metastases can reach 20 $40 \%$. Several studies have demonstrated that the addition of CT to RT improves survival. The EORTC and the RTOG have both recently conducted randomized phase III trials comparing this approach with standard PORT. ${ }^{1,2}$ The EORTC 22931 study showed improved LRC, DFS, and OS with the combined treatment modality. However, the RTOG 10-95 study failed to show a significant difference in terms of OS whereas LRC and DFS were significantly improved by the combined modality. A combined analysis of both studies revealed that addition of concomitant chemotherapy to PORT results in significantly better outcome, especially in patients with inadequate resection margins and/or extranodal spread. ${ }^{6}$

Concomitant CT and hyperfractionated or accelerated RT without surgery has been confirmed by several studies; however, there are no data in the postoperative setting. ${ }^{24-28}$ In our department, accelerated RT has been implemented since 1998. In our experience, concurrent administration of three cycles of full-dose cisplatin and 5-fluorouracil (5-FU) 
TABLE 2 Univariate analyses

\begin{tabular}{|c|c|c|c|c|c|c|c|}
\hline & $\begin{array}{l}N \\
\text { DFS }\end{array}$ & 3-year & $95 \% \mathrm{CI}$ & $P$-value & $\begin{array}{l}\text { 3-year } \\
\text { LRC }\end{array}$ & $95 \% \mathrm{CI}$ & $P$-value \\
\hline All patients & 40 & 62 & $48-78$ & - & 81 & $69-93$ & - \\
\hline \multicolumn{8}{|l|}{ Gender } \\
\hline Female & 4 & 100 & - & 0.13 & 100 & - & 0.29 \\
\hline Male & 36 & 59 & $43-75$ & - & 79 & $65-93$ & - \\
\hline \multicolumn{8}{|l|}{ Age (years) } \\
\hline$<60$ & 20 & 74 & $54-94$ & 0.35 & 90 & $76-100$ & 0.47 \\
\hline$\geq 60$ & 20 & 53 & $31-75$ & - & 73 & $53-93$ & - \\
\hline \multicolumn{8}{|l|}{ Tumor site } \\
\hline Oral cavity & 15 & 63 & $38-87$ & 0.97 & 78 & $56-100$ & 0.95 \\
\hline Oropharynx & 8 & 63 & $30-99$ & - & 75 & $45-100$ & - \\
\hline Larynx & 7 & 71 & $38-104$ & - & 86 & $61-100$ & - \\
\hline Hypopharynx & 8 & 51 & $11-91$ & - & 86 & $61-100$ & - \\
\hline Unknown primary & 2 & - & - & - & - & - & - \\
\hline \multicolumn{8}{|l|}{ T-classification } \\
\hline pT1-2 & 23 & 65 & $46-84$ & 0.85 & 78 & $60-96$ & 0.51 \\
\hline pT3-4 & 17 & 61 & $46-86$ & - & 88 & $72-100$ & - \\
\hline \multicolumn{8}{|l|}{$N$-classification } \\
\hline pN0-1 & 11 & 88 & $64-112$ & 0.04 & 100 & - & 0.07 \\
\hline $\mathrm{pN} 2-3$ & 29 & 55 & $37-73$ & - & 75 & $59-91$ & - \\
\hline \multicolumn{8}{|l|}{ ENE } \\
\hline No & 16 & 66 & $44-96$ & 0.81 & 79 & $68-90$ & 0.96 \\
\hline Yes & 24 & 61 & $41-79$ & & 83 & 67-99 & - \\
\hline \multicolumn{8}{|c|}{ Positive surgical margin } \\
\hline No & 18 & 64 & $41-97$ & 0.92 & 76 & $54-98$ & 0.34 \\
\hline Yes & 22 & 62 & $41-95$ & - & 85 & $69-100$ & - \\
\hline \multicolumn{8}{|l|}{ Surgery-RT interval } \\
\hline$\leq 42$ days & 16 & 56 & $32-80$ & 0.47 & 75 & $53-97$ & 0.56 \\
\hline$>42$ days & 24 & 68 & $48-88$ & - & 86 & $70-100$ & - \\
\hline \multicolumn{8}{|l|}{$R T$ duration } \\
\hline$\leq 39$ days & 21 & 61 & $39-83$ & 0.62 & 80 & $62-98$ & 0.88 \\
\hline$>39$ days & 19 & 66 & $44-88$ & - & 84 & $66-100$ & - \\
\hline
\end{tabular}

TABLE 3 Acute toxicity

\begin{tabular}{lclll}
\hline Toxicity & Grade I $(\%)$ & Grade II $(\%)$ & Grade III $(\%)$ & Grade IV $(\%)$ \\
\hline Mucositis & $11(28)$ & $19(47)$ & $10(25)$ & - \\
Dysphagia & $9(23)$ & $22(54)$ & $9(23)$ & - \\
Erythema & $13(32)$ & $21(53)$ & $6(15)$ & - \\
Anemia & $27(68)$ & $7(18)$ & $1(3)$ & $1(3)$ \\
Leukopenia & $7(18)$ & $3(8)$ & $2(5)$ & $2(5)$ \\
Thrombocytopenia & $12(30)$ & - & - & - \\
Renal toxicity & $10(25)$ & $2(5)$ & $1(3)$ & - \\
\hline
\end{tabular}

CT and concomitant boost accelerated 70-Gy RT is feasible in patients with advanced squamous cell carcinoma of the head and neck. ${ }^{29}$ We also reported our results using accelerated PORT, in which 2-year actuarial LRC was $80 \%$, and distant metastases probabilty was $38 \%$ with acceptable toxicity. ${ }^{11}$ In the present study, we aimed to assess whether weekly concomitant boost (six fractions per week) accelerated PORT combined with cisplatin CT is better than accelerated PORT alone. Three-year OS and LRC rates were $65 \%$ and $82 \%$, respectively; distant 
metastases probability at 3 years was $19 \%$. The incidence of grade 3 mucositis was $25 \%$, grade 3 dysphagia $23 \%$, and grade 3 skin erythema $13 \%$. CT-related bone marrow toxicity was observed in six patients (15\%), and grade 3 renal function impairment was observed in only one patient. In this study, $95 \%$ of patients received the prescribed full dose of radiation, and only $54 \%$ of patients were able to complete the full cisplatin dose (three cycles) with moderate toxicity, a finding also observed in the EORTC and RTOG studies. ${ }^{1,2}$ In the future, it should be considered whether or not to continue with this third cycle of cisplatin. Acceptable toxicity rates in our series are most likely due to our relatively soft accelerated RT schedule. Treating the boost volume every Friday afternoon during the first 5 weeks by using smaller PTVs is easily accepted by patients as well as the radiotherapy department.

The results of this trial demonstrated that reducing the overall treatment time using accelerated PORT/CT by weekly concomitant boost (six fractions per week) combined with concomitant cisplatin CT is easily feasible with good locoregional and distant metastases control in curatively operated patients with LAHNC. However, acute toxicity remains the main obstacle to wide acceptance of this treatment modality requiring close follow-up and supportive care by an experienced multidisciplinary team. Current research strategies will continue to focus on improving the therapeutic ratio in an attempt to achieve higher rates of both local and systemic control. New targeted therapy agents with less toxicity should be investigated, combined with current chemoradiation regimens.

\section{REFERENCES}

1. Bernier J, Domenge C, Ozsahin M, Matuszewska K, Lefèbvre JL, Greiner RH, et al. Postoperative irradiation with or without concomitant chemotherapy for locally advanced head and neck cancer. $N$ Engl J Med. 2004;350:1945-52.

2. Cooper JS, Pajak TF, Forastiere AA, Jacobs J, Campbell BH, Saxman SB, et al. Postoperative concurrent radiotherapy and chemotherapy for high-risk squamous-cell carcinoma of the head and neck. N Engl J Med. 2004;350:1937-44.

3. Zelefsky MJ, Harrison LB, Fass DE, Armstrong JG, Shah JP, Strong EW. Postoperative radiation therapy for squamous cell carcinomas of the oral cavity and oropharynx: impact of therapy on patients with positive surgical margins. Int J Radiat Oncol Biol Phys. 1993;25:17-21.

4. Amdur RJ, Parsons JT, Mendenhall WM, Million RR, Cassisi NJ. Postoperative irradiation for squamous cell carcinoma of the head and neck: an analysis of treatment results and complications. Int J Radiat Oncol Biol Phys. 1989;16:25-36.

5. Tupchong L, Scott CB, Blitzer PH, Marcial VA, Lowry LD, Jacobs JR, et al. Randomized study of preoperative versus postoperative radiation therapy in advanced head and neck carcinoma: Long-term follow-up of RTOG study 73-03. Int J Radiat Oncol Biol Phys. 1991;20:21-8.

6. Bernier J, Cooper JS, Pajak TF, van Glabbeke M, Bourhis J, Forastiere A, et al. Defining risk levels in locally advanced head and neck cancers: a comparative analysis of concurrent postopeartive radiation plus chemotherapy trials of the EORTC 22931 and RTOG 9501. Head Neck. 2005;27:843-50.

7. Bernier J. Current state-of-the-art for concurrent chemoradiation. Semin Radiat Oncol. 2009;19:3-10.

8. Peters LJ, Withers HR. Applying radiobiological principles to combined modality treatment of head and neck cancer-the time factor. Int J Radiat Oncol Biol Phys. 1997;39:831-6.

9. Fu KK, Pajak TF, Trotti A, Jones CU, Spencer SA, Phillips TL, et al. A Radiation Therapy Oncology Group (RTOG) phase III randomized study to compare hyperfractionation and two variants of accelerated fractionation to standard fractionation radiotherapy for head and neck squamous cell carcinomas: first report of RTOG 9003. Int J Radiat Oncol Biol Phys. 2000;48:7-16.

10. Kajanti MJ, Holsti LR. Radical surgery and postoperative splitcourse radiotherapy in squamous cell carcinoma of the head and neck-factors influencing local control and survival. Acta Oncol. 1993;32:319-25.

11. Zouhair A, Azria D, Pasche P, Stupp R, Chevalier J, Betz M, et al. Accelerated postoperative radiotherapy with weekly concomitant boost in patients with locally advanced head and neck cancer. Radiother Oncol. 2004;70:183-8.

12. Head and neck tumours. International Union Against Cancer (UICC), In: Sobin LH, Wittekind Ch,editors. TNM classification of malignant tumours, 6th ed. New York:Wiley-Liss; 2002. p.19-47.

13. Piquet MA, Ozsahin M, Larpin I, Zouhair A, Coti P, Monney M, et al. Early nutritional intervention in oropharyngeal cancer patients undergoing radiotherapy. Support Care Cancer. 2002;10:502-4.

14. Kaplan EL, Meier P. Non parametric estimation from incomplete observations. J Am Stat Assoc. 1958;53:457-81.

15. Peto R, Pike MC, Armitage P, Breslow NE, Cox DR, Howard $\mathrm{SV}$, et al. Design and analysis of randomised clinical trials requiring prolonged observation of each patients: part II. $\mathrm{Br} \mathrm{J}$ Cancer. 1977;35:1-39.

16. Cox DR. Regression models and life tables. $J$ R Stat Soc. 1972;34:187-220.

17. Bernier J. Alteration of radiotherapy fractionation and concurrent chemotherapy: a new frontier in head and neck oncology? Nature Clin Practice Oncol. 2005;2:305-14.

18. Horiot JC, Le Fur R, N'Guyen T, Chenal C, Schraub S, Alfonsi S, et al. Hyperfractionation versus conventional fractionation in oropharyngeal carcinoma: fnal analysis of a randomized trial of the EORTC cooperative group of radiotherapy. Radiother Oncol. 1992;25:231-40.

19. Bourhis J, Overgaard J, Audry H, Ang KK, Saunders M, Bernier $\mathrm{J}$, et al. Hyperfractionated or accelerated radiotherapy in head and neck cancer: a meta-analysis. Lancet. 2006;368:843-54.

20. Ang KK, Trotti A, Brown BW, Garden AS, Foote RL, Morrison $\mathrm{WH}$, et al. Randomized trial addressing risk features and time factors of surgery and radiotherapy in advanced head and neck cancer. Int J Radiat Oncol Biol Phys. 2001;51:571-78.

21. Overgaard J, Hansen HS, Specht L, Overgaard M, Grau C, Andersen E, et al. Five compared with six fractions per week of conventional radiotherapy of squamous-cell carcinoma of head and neck: DAHANCA 6\&7 randomised controlled trial. Lancet. 2003;362:933-40.

22. Sanguineti G, Richetti A, Bignardi M, Corvo' R, Gabriele P, Sormani MP, et al. Accelerated versus conventional fractionated postoperative radiotherapy for advanced head and neck cancer: results of a multicenter phase III study. Int J Radiat Oncol Biol Phys. 2005;61:762-71.

23. Suwiński R, Bańkowska-Woźniak M, Majewski W, Idasiak A, Maciejewski A, Ziółkowska E, et al. Randomized clinical trial on continuous 7-days-a-week postoperative radiotherapy for 
high-risk squamous cell head-and-neck cancer: a report on acute normal tissue reactions. Radiother Oncol. 2006;80:27-32.

24. Brizel DM, Albers ME, Fisher SR, Scher RL, Richtsmeier WJ, Hars V, et al. Hyperfractionated irradiation with or without concurrent chemotherapy for locally advanced head and neck cancer. N Engl J Med. 1998;338:1798-804.

25. Jeremic B, Shibamoto Y, Milicic B, Nikolic N, Dagovic A, Aleksandrovic J, et al. Hyperfractionated radiation therapy with or without concurrent low-dose daily cisplatin in locally advanced squamous cell carcinoma of the head and neck: a prospective randomized trial. J Clin Oncol. 2000;18:1458-64.

26. Staar S, Rudat V, Stuetzer H, Dietz A, Volling P, Schroeder M, et al. Intensified hyperfractionated accelerated radiotherapy limits the additional benefit of simultaneous chemotherapy: results of a multicentric randomized German trial in advanced head-and-neck cancer. Int J Radiat Oncol Biol Phys. 2001;50:1161-71.
27. Huguenin P, Beer KT, Allal A, Rufibach K, Friedli C, Davis JB, et al. Concomitant cisplatin significantly improves locoregional control in advanced head and neck cancers treated with hyperfractionated radiotherapy. J Clin Oncol. 2004;22:4665-73.

28. Budach V, Stuschke M, Budach W, Baumann M, Geismar D, Grabenbauer G, et al. Hyperfractionated accelerated chemoradiation with concurrent fluorouracil-mitomycin is more effective than dose-escalated hyperfractionated accelerated radiation therapy alone in locally advanced head and neck cancer: final results of the radiotherapy cooperative clinical trials group of the German Cancer Society 95-06 Prospective Randomized Trial. J Clin Oncol. 2005;23:1125-35.

29. Kutter J, Ozsahin M, Monnier P, Stupp R. Combined modality treatment with full-dose chemotherapy and concomitant boost radiotherapy for advanced head and neck carcinoma. Eur Arch Otorhinolayngol. 2005;262:1-7. 